\title{
A Time-Series Analysis of Severe Burned Injury of Skin Gene Expression Profiles
}

\author{
Hai-Ting Xua Jian-Chun Guo ${ }^{c}$ Hua-Zhen Liud Wan-Wan Jin ${ }^{b}$ \\ aDepartment of Plastic Surgery, The Second Affiliated Hospital and Yuying Children's Hospital of \\ Wenzhou Medical University, Wenzhou, bepartment of Dermatology, The Second Affiliated Hospital \\ and Yuying Children's Hospital of Wenzhou Medical University, Wenzhou, 'School of Laboratory \\ Medicine and Life Sciences, Wenzhou Medical University, Wenzhou, dDepartment of Burn Surgery, \\ Changhai Hospital, Second Military Medical University, Shanghai, China
}

\author{
Key Words \\ Geo $\cdot$ Burned $\cdot$ Soft clustering $\cdot \mathrm{DAVID} \cdot \mathrm{R}$
}

\begin{abstract}
Background/Aims: Major burn injury is one of the main severe forms of wound which lead to a mass of clinical debilitation, this study was to identify core biomarkers for the recovery of severe burned injury. Methods: Gene expression profiles (GSE19743) from the Gene Expression Omnibus (GEO) was downloaded, followed by background correction, normalization of raw microarray dataset and identification of differential expression genes (DEGs). Soft clustering of DEGs was used for the screening of gene clusters that with sustained increasing (SIG) and decreasing expression (SDG) profiles along with the recovery process of burned injury. The significantly enriched Gene Ontology (GO) terms and Kyoto Encyclopedia of Genes and Genomes (KEGG) pathways of SIGs and SDGs were obtained through the Database for Annotation, Visualization, and Integrated Discovery (DAVID), based on which the miRNAgene regulation network for SIGs and SDGs were constructed from the miRWalk database. Results: Ten clusters were obtained through soft clustering. The SIGs and SDGs were found to be closely associated with the biological processes of immune system. The miRNA-gene regulation network analysis suggested different roles between SIGs and SDGs in the recovery of severe burned injury. Furthermore, a bunch of important biomarkers were identified, which would be helpful in the treatment of burned patients. Conclusion: Our current findings suggest an interesting molecular link between transcriptional regulation potentially involved in immunosuppressive state after major burn injury, which warrants further exploration for their utilization in the treatment of major burn injury.
\end{abstract}




\section{Cellular Physiology Cell Physiol Biochem 2018;49:1492-1498 and Biochemistry \begin{tabular}{l|l} 
DOI: 10.1159/000493451 \\
Published online: 13 September, 2018 & $\begin{array}{l}\text { O } 2018 \text { The Author(s). Published by S. Karger AG, Basel } \\
\text { www.karger.com/cpb }\end{array}$
\end{tabular} \\ Xu et al.: A Time-Series Analysis of Severe Gene Expression Profiles}

\section{Introduction}

Major burn injury is one of the main severe forms of wound which lead to a mass of clinical debilitation coupled with economic influence, influencing up to millions of injuries and deaths per year in the world. In addition to the devastation of the skin barriers protection by major burn injury, one of the most complications involving the clinical course of recovery is generalized immunocompromise that further caused recurrent inflammation and prolonged healing. Twenty years ago, Wolfe et al. noticed that immunosuppressive serum and an impaired lymphocyte response are associated with anergy in burn patients and confirm that the development of anergy is an index of poor prognosis [1]. Then, several attempts have been made to clarify the relationship of immunosuppressive and burn injury. Barrow and his colleague found that burn injury induced a significant increase in serum TGF-beta, which may contribute to post-burn immunosuppression with an increased susceptibility to sepsis [2]. Another study have demonstrated that the productive capacity of macrophages for inflammatory mediators, including nitric oxide, prostaglandins, TNF- $\alpha$ and $I L-6$, is profoundly increased post-burn, thereby implicating macrophages in the development of the post-burn immunosuppression [3]. What's more, production of interleukin-10 has also been shown to be increased by cells of the immune system in human and mouse after burn injury [4]. All of these studies indicated the importance to explore the mechanisms of immunosuppressive and burn injury. Despite recognized roles for immunosuppressive in regulating healing and infections of burn injury, our mechanistic understanding of immunocompromise and its systemic influence, and our capacity to manipulate this response are still limited.

In this study, gene expression profiles (GSE19743) were downloaded from the Gene Expression Omnibus (GEO). They collected leukocytes from blood samples of severe burned patients and healthy controls at different times after severe burn injury. Total cellular RNA was isolated from the leukocyte pellets and gene expression was measured using Affymetrix U133 Plus 2.0 arrays. Background correction, normalization of raw microarray dataset and identification of differential expression genes (DEGs) were conducted via R. Soft clustering of DEGs was used for the screening of gene clusters that with sustained increasing (SIG) and decreasing expression (SDG) profiles with the recovery of burned injury. What's more, SIGs and SDGs were uploaded to David online database to identify significantly enriched GO terms and KEGG pathways. Then we focus on genes associated with transcription control. In conclusion, our finding has explained the central importance of understanding immunosuppressive state after major burn injury and provide potential therapeutic targets for further studies.

\section{Materials and Methods}

\section{Gene Expression Datasets}

We obtained the gene expression profiles from the Gene Expression Omnibus (GEO, https://www.ncbi. nlm.nih.gov/geo/) in NCBI with the accession number of GSE19743[5]. A total of 177 blood samples of 57 severe burned patients with two biological replicates and 63 healthy controls with one replicate were contained in the dataset. In this study, we divided the samples into four groups, i.e. healthy controls, patients of $0 \sim 100$ hours, 100 400 hours and longer than 400 hours after severe burn injury, and they were referred as ' $\mathrm{H}$ ', 'E', 'M' and 'L' respectively hereafter.

\section{Microarray Analysis}

All of the analysis processes of the gene expression microarray were conducted in R. Briefly, raw microarray datasets in CEL were imported into R and a Robust Multi-Array (RMA) method was adopted for the background correction and normalization through affy package [6]. Then, we identified the differential expression genes (DEGs) in the 114 burned patients compared with the 63 healthy controls via limma package [7] with the criteria of fold change $>2$ and FDR adjusted $P$-Value $<0.05$. 


\section{Cellular Physiology Cell Physiol Biochem 2018;49:1492-1498 \begin{tabular}{l|l} 
DOI: 10.1159/000493451 & $\begin{array}{l}\text { O 2018 The Author(s). Published by S. Karger AG, Basel } \\
\text { www.karger.com/cpb }\end{array}$
\end{tabular}

\section{Soft Clustering of DEGs}

We considered genes with sustained increasing or decreasing expression profiles with the recovery of injury as those important ones. So one of the core processes is to identify those genes. In this study, we conducted soft clustering through Mfuzz package [8], which could allocate a gene into multi clusters according to its clustering coefficient (CC). Here, we screened out the gene clusters with sustained increasing and decreasing profiles from $\mathrm{H}$ group to L group, i.e. $\mathrm{H}-\mathrm{E}-\mathrm{M}-\mathrm{L}$ through the soft clustering of DEGs.

\section{Functional Enrichment Analysis}

To explore the enriched functions of genes in sustained increasing (SIG) and decreasing (SDG) clusters, functional enrichment analysis was conducted in the Database for Annotation, Visualization and Integrated Discovery (DAVID, https://david.ncifcrf.gov/)[9]. Gene Ontology (GO) terms and Kyoto Encyclopedia of Genes and Genomes (KEGG) pathways with P-Value $<0.05$ were obtained.

\section{MiRNA-Gene Regulation Network}

MicroRNA (miRNA) is a small non-coding RNA molecule containing about 22 nucleotides. Through regulating the expression of its target genes, it could affect the progression of many types of diseases, such as cancer. While very few studies have focused on the influences of miRNA in the recovery of burned injury. In this study, we screened out miRNAs that might regulate SIGs and SDGs through miRWalk database [10]. Only the miRNA-gene pairs exist in all of the TargetScan, miRanda, RNA22, PITA and miRWalk databases were considered reliable. Besides, the miRNA-gene regulation network was visualized through Cytoscape [11].

\section{Results}

\section{Differential Expression Analysis}

The normalized expression values were used for the identification of DEGs. With the criteria of fold change $>2$ and FDR adjusted P-Value $<0.05$, a total of 1215 DEGs were identified in the 114 burned samples compared with the 63 healthy controls.

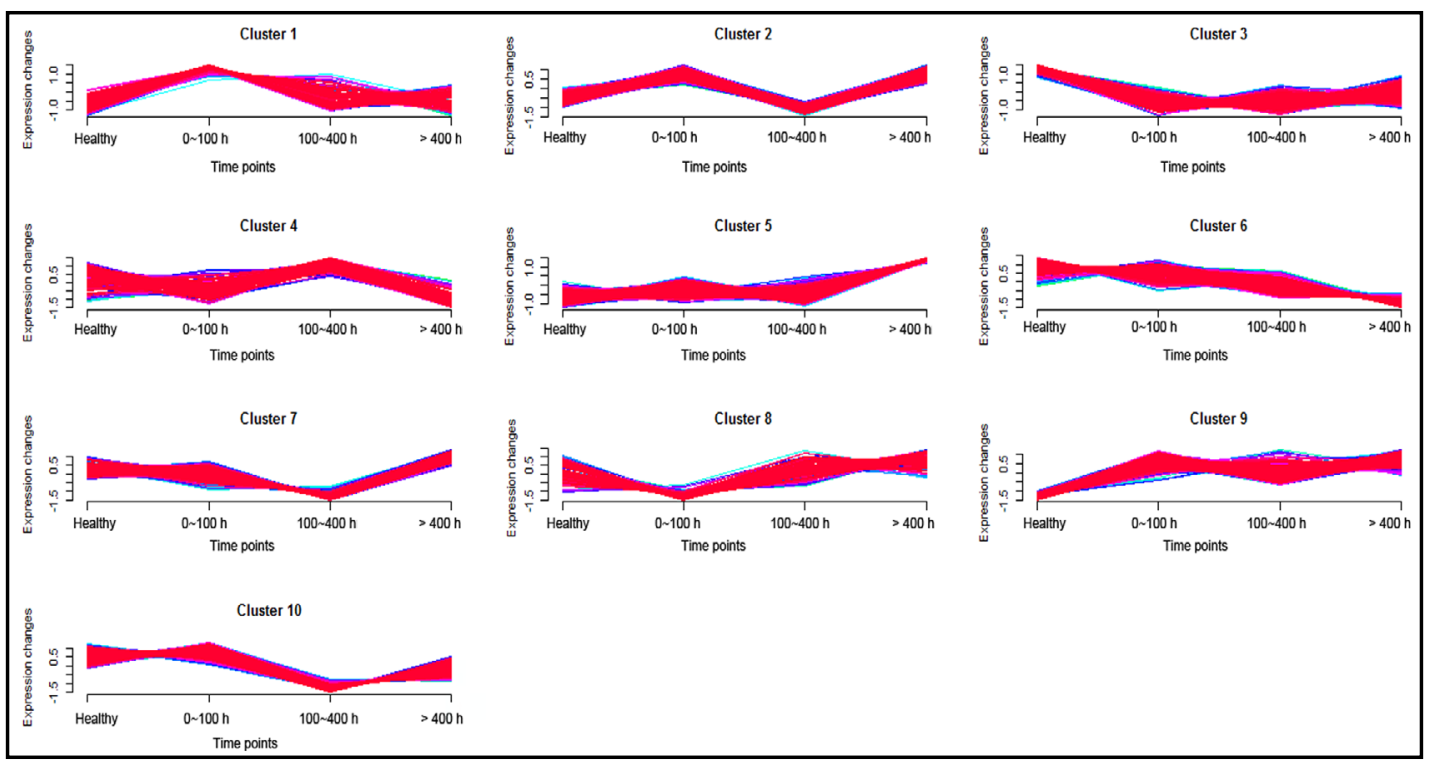

Fig. 1. The 10 clusters obtained through soft clustering. 


\section{Cellular Physiology Cell Physiol Biochem 2018;49:1492-1498 \begin{tabular}{ll|l} 
DOI: 10.1159/000493451 & $\begin{array}{l}\text { O } 2018 \text { The Author(s). Published by S. Karger AG, Basel } \\
\text { www.karger.com/cpb }\end{array}$ \\
\hline and Biochemistry
\end{tabular} \\ Xu et al.: A Time-Series Analysis of Severe Gene Expression Profiles}

\section{SIGs and SDGs}

Soft clustering of the 1215 DEGs resulted in 10 clusters (Fig. 1), and among which, most of the genes in cluster 6 were found to be with sustained decreasing expression profiles from $\mathrm{H}$ to $\mathrm{L}$ and the genes in cluster 8 with sustained increasing expression profiles. Thus, genes in cluster 6 and cluster 8 with CC $>0.5$ were considered as SDGs and SIGs respectively. The top 10 SDGs and SIGs with the highest score in cluster 6 and cluster 8 were shown in Table 1 .

For other clusters, for example cluster 1 and cluster 2, whose expression profiles were firstly upregulated followed by down-regulated. Meanwhile, some clusters have inverse expression regulation trend, for example cluster 3 and cluster 4 , in which the gene expression values were firstly down-regulated followed by up-regulated.
Table 1. Top 10 genes with the highest scores in cluster 6 and cluster 8

\begin{tabular}{lccc}
\hline Cluster 6 & Score & Cluster8 & Score \\
\hline LMO7DN & 1.000 & DPEP2 & 0.999 \\
CISD3 & 1.000 & NOG & 0.999 \\
ADAM28 & 0.999 & CBFA2T3 & 0.999 \\
ABCB1 & 0.999 & ZNF76 & 0.999 \\
TRPC1 & 0.999 & TRPM2 & 0.995 \\
MRPS25 & 0.999 & ADGRE3 & 0.995 \\
QDPR & 0.999 & ALKBH2 & 0.993 \\
MS4A1 & 0.997 & ANKRD23 & 0.992 \\
CARNS1 & 0.994 & WDR60 & 0.992 \\
E2F2 & 0.993 & B3GAT1 & 0.990 \\
\hline
\end{tabular}

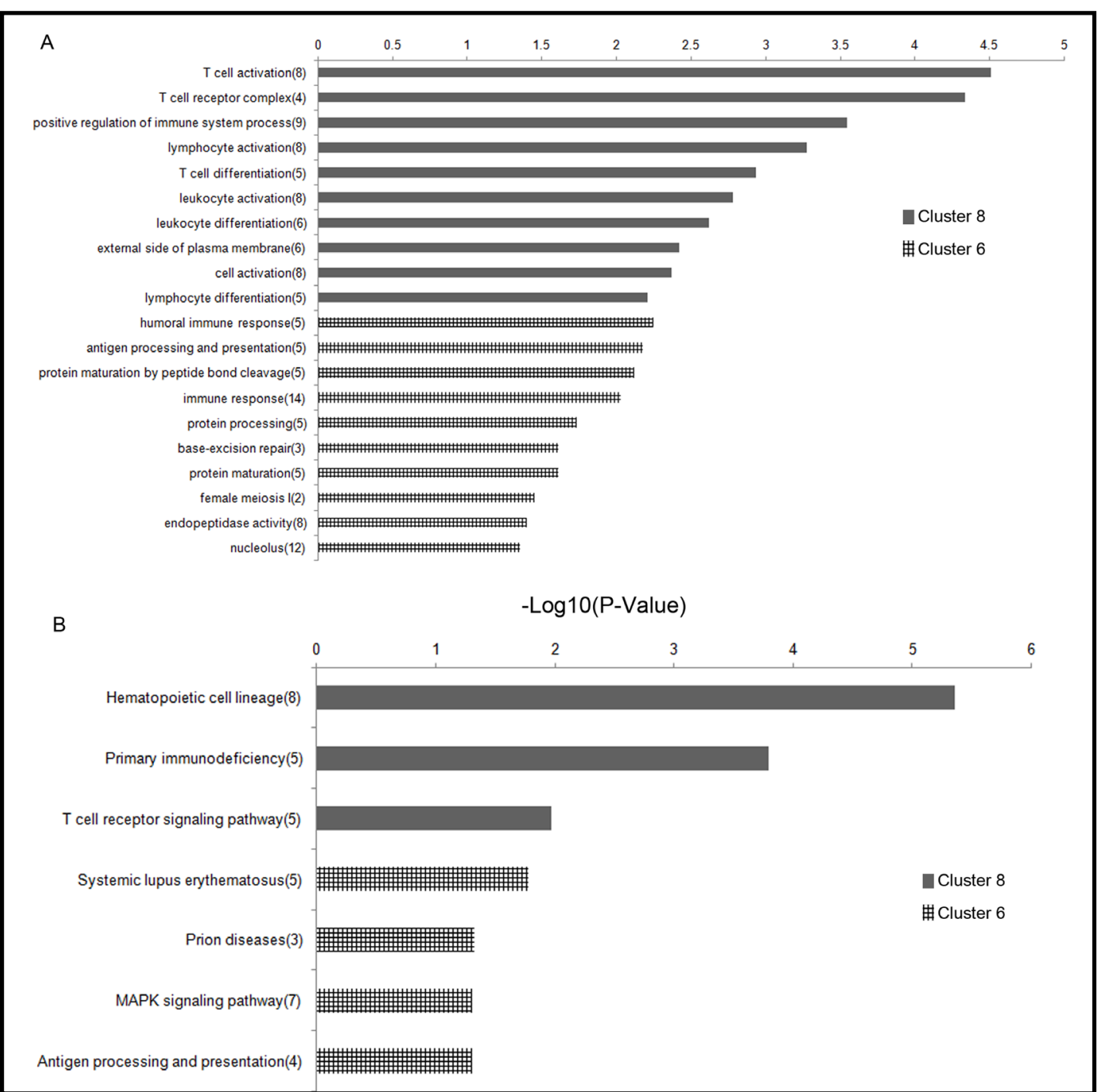

Fig. 2. The top 10 most significant GO terms (A) and all of the significantly enriched KEGG pathways (B) for SIGs and SDGs. 


\section{Cellular Physiology Cell Physiol Biochem 2018;49:1492-1498

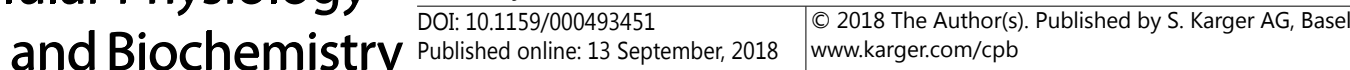

\section{Enriched Functions}

A total of 89 and 76 enriched GO terms were obtained for SDGs and SIGs respectively with the criteria of P-Value < 0.05 . Most of those terms were found to be associated with immune response, such as $\mathrm{T}$ cell activation, $\mathrm{T}$ cell complex receptor, humoral immune response, antigen processing and presentation. Besides, there were four and three KEGG pathways were significantly enriched in SIGs and SDGs respectively, which also mainly involved in the biological processes of immune system. Fig. 2A and 2B illustrated the top 10 significantly enriched GO terms and all of the KEGG pathways for SIGs and SDGs.

\section{MiRNA-Gene Regulation Network}

The miRNA-gene regulation networks for SDGs and SIGs were shown in Supplemental Fig. S1 and Supplemental Fig. S2 respectively (For all supplemental material see www. karger.com/10.1159/000493451/). The distribution of degree (number of nodes directly link with one node) of the two miRNA-gene regulation network were shown in Fig. $3 \mathrm{~A}$ and $\mathrm{B}$ and compared through wilcoxon test. As a result, degrees in the miRNA-gene regulation network of SIGs were significantly higher than that of SIGs' (Fig. 3C, $P$-Value $=0.0485$ ). Besides, Table 2 showed the top 10 miRNAs with highest degree in the two networks.

\section{Discussion}

In the past two decades, a number of researchers have reported that major burn injury causes an immunosuppressive state that predisposes victims to subsequent sepsis and multiple organ failure. A considerable amount of literature has been published on immunosuppressive mechanisms, including dominance of inhibitory over activating receptors, expansion of suppressive cell types, as well as proinflammatory and anti-inflammatory cytokines injury. However, far too little attention has been paid to transcriptional regulation of immunosuppressive state in major burn injury study. The fungal metabolites cyclosporin A (CsA) and tacrolimus (FK506),
Table 2. The top 10 miRNAs with the largest number of targets in the miRNA-Gene network of cluster 6 and cluster 8

\begin{tabular}{|c|c|c|c|}
\hline Cluster 6 & Degree & Cluster8 & Degree \\
\hline miR-325-3p & 17 & miR-23-3p & 12 \\
\hline miR-29 & 16 & $\begin{array}{c}\text { miR-181-5 } \\
\text { p }\end{array}$ & 11 \\
\hline miR-29-3p & 16 & miR-506 & 10 \\
\hline miR-29bc & 16 & $\begin{array}{c}\mathrm{miR}-340-5 \\
\mathrm{p}\end{array}$ & 10 \\
\hline miR-124 & 15 & $\begin{array}{c}\text { miR-325-3 } \\
\text { p }\end{array}$ & 10 \\
\hline $\begin{array}{l}\mathrm{miR}-29 \mathrm{bc}-3 \\
\mathrm{p}\end{array}$ & 14 & miR-27-3p & 10 \\
\hline $\operatorname{miR}-27$ & 13 & $\operatorname{miR}-25$ & 10 \\
\hline $\operatorname{miR}-27-3 p$ & 13 & miR-23 & 10 \\
\hline miR-302 & 13 & miR-204 & 10 \\
\hline miR-23-3p & 12 & miR-181 & 10 \\
\hline
\end{tabular}

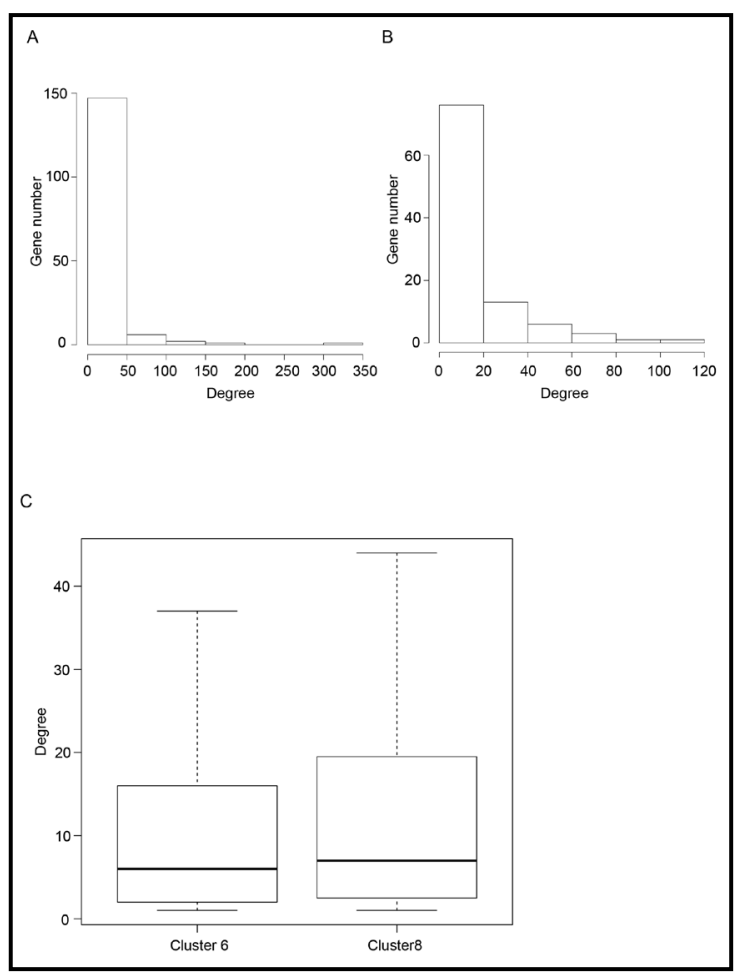

Fig. 3. The degree distribution of miRNA-gene network of SIGs (A) and SDGs (B) and the comparison of those two lists of degrees (C). 


\section{Cellular Physiology Cell Physiol Biochem 2018;49:1492-1498 \begin{tabular}{l|l} 
DOI: 10.1159/000493451 & $\begin{array}{l}\text { O } 2018 \text { The Author(s). Published by S. Karger AG, Basel } \\
\text { www.karger.com/cpb }\end{array}$
\end{tabular} \\ Xu et al.: A Time-Series Analysis of Severe Gene Expression Profiles}

which inhibit the calcium-dependent serine/threonine phosphatase calcineurin and its substrate, the transcription factor NFAT, are among the most potent immunosuppressive drugs available today. In addition of $N F A T$, a number of transcription factors have reported to control immunosuppression. For example, transcription factors Stat3 and Gfi-1 could control Th17 cell immunosuppressive state by induced the expression of ectonucleotidases. What's more, TGF- $\beta$ induced Foxp3 gene expression in TCR-challenged CD4+CD25- naive $\mathrm{T}$ cells, which mediated their transition toward a regulatory $\mathrm{T}$ cell phenotype with potent immunosuppressive potential. All of these researches implied that transcription factor may be potent drug target for immunosuppressive therapy.

To understand the cell and molecule mechanism response to burn injury, leukocytes from blood samples of severe burned patients and healthy controls were collected at different times after severe burn injury. After background correction, normalization of raw microarray dataset and identification of differential expression genes (DEGs), a total of 1215 DEGs were identified. Soft clustering of DEGs was used for the screening of gene clusters that with SIG and SDG profiles with the recovery of burned injury. The significantly enriched GO terms and KEGG pathways of SIGs and SDGs were obtained through DAVID online database. In accordance with other research, significantly enriched ontology categories were found to be closely associated with immune activation, including immune response, $\mathrm{T}$ cell differentiation, $\mathrm{T}$ cell costimulation, adaptive immune response and positive regulation of calcium-mediated signaling and etc. Interestingly, there is another GO term associated with transcriptional control drawn our attention. We noticed that the expression level of some transcription factors, including ELP2, FOXO6, ZNF18, ZNF76, POU3F4, TEAD3 and $S U P T 3 H$ were sustained increased post burn injury. It is reasonable to believe that the up-regulation of these transcription factors maybe drivers or at least played a critical for inducing the immunosuppressive state. ELP2 is a core subunit of the elongator complex effected transcriptional elongation and may help remodel chromatin. Mou and his colleagues showed that Elongator subunit 2 (ELP2) regulated the kinetics of defense gene induction and accelerated immune responses [12]. FOXO6 is an important paralog of FOXO1. Recent studies have highlighted a fundamental role for Forkhead box 0 (FOXO) transcription factors in immune system homeostasis [13]. We believe that these genes may be critical mediators in pathogenesis of immunocompromise which finally manipulate poor prognosis.

In this study, we also conducted miRNA-gene regulation analysis for SIGs and SDGs. MiR-23-3p has the most SDGs targets than other miRNAs in miRNA-gene regulation network of SDGs. In Pedrera's study [14], miR-23-3p was found to be significantly up-regulated by anti-TNF $\alpha$ in rheumatoid arthritis patients, which indicated its role in immune response. Here, we demonstrated that post-burn immunosuppression was proved to contribute burn recovery, so miR-23-3p might also involve in the biological processes post-burn. MiR-29 is the second miRNA with the most gene targets in miRNA-gene regulation network of SIGs. There are several studies indicated the important role of miR-29 in immune response [1517], so miR-29 should also be an important biomarker for burn recovery. It is important to take miRNA into account for the discovery of novel drugs for severe burn treatment.

\section{Conclusion}

All in all, by the identification of DEGs, clustering, GO enrichment, KEGG pathway analysis, some key genes that might play important roles in immunocompromise were screened out. And our current findings suggest an interesting molecular link between transcriptional regulation potentially involved in immunosuppressive state after major burn injury. However, the results of our study remains to be further explored for their utilization in the treatment of major burn injury. 


\section{Cellular Physiology Cell Physiol Biochem 2018;49:1492-1498 \begin{tabular}{ll|l} 
and Biochemistry & $\bar{D}$ DoI: 10.1159/000493451 & $\begin{array}{l}\text { Published online:13 } 2018 \text { The Author(s). Published by S. Karger AG, Basel } \\
\text { www.karger.com/cpb }\end{array}$ \\
\cline { 2 - 3 }
\end{tabular}

\section{Disclosure Statement}

The authors declare to have no competing interests.

\section{References}

1 Wolfe JH, Wu AV, O’Connor NE, Saporoschetz I, Mannick JA: Anergy, immunosuppressive serum, and impaired lymphocyte blastogenesis in burn patients. Arch Surg 1982;117:1266-1271.

-2 Varedi M, Jeschke MG, Englander EW, Herndon DN, Barrow RE: Serum TGF-beta in thermally injured rats. Shock 2001;16:380-382.

-3 Schwacha MG, Chaudry IH: The cellular basis of post-burn immunosuppression: macrophages and mediators. J Mol Med 2002;10:239-243.

4 Lyons A, Kelly JL, Rodrick ML, Mannick JA, Lederer JA: Major injury induces increased production of interleukin-10 by cells of the immune system with a negative impact on resistance to infection. Ann Surg 1997;226:450-458; discussion 458-460.

5 Zhou B, Xu W, Herndon D, Tompkins R, Davis R, Xiao W, Wong WH, Inflammation and Host Response to Injury Program, Toner M, Warren HS, Schoenfeld DA, Rahme L, McDonald-Smith GP, Hayden D, Mason P, Fagan S, Yu YM, Cobb JP, Remick DG, Mannick JA, Lederer JA, et al.: Analysis of factorial time-course microarrays with application to a clinical study of burn injury. Proc Natl Acad Sci U S A 2010;107:99239928.

6 Gautier L, Cope L, Bolstad BM, Irizarry RA: affy--analysis of Affymetrix GeneChip data at the probe level. Bioinformatics 2004;20:307-315.

7 Diboun I, Wernisch L, Orengo CA, Koltzenburg M: Microarray analysis after RNA amplification can detect pronounced differences in gene expression using limma. BMC Genomics 2006;7:252.

-8 Kumar L, E Futschik M: Mfuzz: a software package for soft clustering of microarray data. Bioinformation 2007;2:5-7.

9 Dennis G Jr, Sherman BT, Hosack DA, Yang J, Gao W, Lane HC, Lempicki RA: DAVID: Database for Annotation, Visualization, and Integrated Discovery. Gen Biol 2003;4:P3.

10 Dweep H, Sticht C, Pandey P, Gretz N: miRWalk--database: prediction of possible miRNA binding sites by "walking" the genes of three genomes. J Biomed Inform 2011;44:839-847.

11 Paul S, Andrew M, Owen O, Nitin S. B, Jonathan TW, Daniel R, Nada A, Benno S, Trey I: Cytoscape: a software environment for integrated models of biomolecular interaction networks. Genome Res 2003;13:24982504.

12 DeFraia CT, Zhang X, Mou Z: Elongator subunit 2 is an accelerator of immune responses in Arabidopsis thaliana. Plant J 2010;64:511-523.

13 Dejean AS, Hedrick SM, Kerdiles YM: Highly specialized role of Forkhead box 0 transcription factors in the immune system. Antioxid Redox Signal 2011;14:663-674.

14 Castro-Villegas C, Pérez-Sánchez C, Escudero A, Filipescu I, Verdu M, Ruiz-Limón P, Aguirre MA, JiménezGomez Y, Font P, Rodriguez-Ariza A, Peinado JR, Collantes-Estévez E, González-Conejero R, Martinez C, Barbarroja N, López-Pedrera C: Circulating miRNAs as potential biomarkers of therapy effectiveness in rheumatoid arthritis patients treated with anti-TNFalpha. Arth Res Ther 2015;17:49.

15 Schmitt MJ, Margue C, Behrmann I, Kreis S: MiRNA-29: a microRNA family with tumor-suppressing and immune-modulating properties. Curr Mol Med 2013;13:572-585.

16 Ma F, Xu S, Liu X, Zhang Q, Xu X, Liu M, Hua M, Li N, Yao H, Cao X: The microRNA miR-29 controls innate and adaptive immune responses to intracellular bacterial infection by targeting interferon-gamma. Nature Immunol 2011;12:861-869.

17 Liston A, Papadopoulou AS, Danso-Abeam D, Dooley J: MicroRNA-29 in the adaptive immune system: setting the threshold. Cell Mol Life Sci 2012;69:3533-3541. 